\title{
Complex Network Construction and Pattern Recognition of China's Provincial Low-Carbon Economic Development with Long Time Series: Based on the Detailed Spatial Relationship
}

\author{
Zeying Lan', Xiaohui Chen ${ }^{1 *}$, Yang $\mathrm{Liu}^{2}$, Dequan Chen ${ }^{3}$, Weiqi $\mathrm{Li}^{4}$ \\ ${ }^{1}$ School of Management, Guangdong University of Technology, Guangzhou 510520, China \\ ${ }^{2}$ Guangzhou Urban Planning \& Design Survey Research Institute, Guangzhou 510060, China \\ ${ }^{3}$ Guangdong Marine Development Planning Research Center, Guangzhou 510220, China \\ ${ }^{4}$ Urumqi Land Reserve Center (Urumqi Land Consolidation Center), Urumqi 830091, China
}

Received: 23 May 2021

Accepted: 23 October 2021

\begin{abstract}
Low-carbon economic development has become the orientation of high-quality economic construction in the era of climate change. Because it involves multi-dimensional elements and is driven by the concept of regional integration and common development, the development pattern among China's provinces should show the characteristics of complex networked spatial correlation. However, most of the existing research are for is based on attribute data and combined with traditional spatial econometric models, which cannot describe the complex networked spatial correlation. Concurrently, the network construction is mainly based on undirected-unweighted sparseness, which makes it difficult to truly restore the asymmetric detailed spatial relationship, making the related research about development pattern recognition and its evolution based on the detailed spatial relationship relatively lacking. Therefore, based on the perspective of relational data, this study constructs a multi-dimensional gravity model by using the comprehensive quality of China's provincial low-carbon economic development, measured by the multi-dimensional evaluation index system and dynamic weight method, and the comprehensive distance based on geography, society, economy, and adjacency. And then the spatial correlation strength among China's provincial low-carbon economic development is determined, and constructs a directed-weighted complex network. Then, it further explores the inter-provincial detailed spatial relationship of low-carbon economy development from the three dimensions of overall, individual and group, to recognize the development patterns and evolution rules of low-carbon economic development in different provinces, and clarify the development orientation. The results show that, during the study period, the spatial correlation of China's provincial
\end{abstract}

*e-mail: 1480593328@qq.com 
low-carbon economic development has become increasingly close, showing a complex networked correlation structure with multi-linearity, asymmetry, and geographical proximity as a whole. The division of general structure is consistent with China's regional economic development strategy. The unbalanced development of China's provincial low-carbon economy is the result of multi-center drive in the eastern and central regions, and the distance from the network center in the western regions. In general, China's provincial low-carbon economic development patterns can be roughly divided into spillover, main bridge, auxiliary bridge, and benefit type. In the future, we should make full use of the power source role of spillover provinces, and the bridge support role of bridge provinces to drive the development of benefit provinces. Among them, Chongqing, Shaanxi, Hebei and Sichuan are expected to become new regional growth poles. The research on the inter-provincial spatial correlation effect and development pattern recognition of low-carbon economic development is expected to provide guidance for the sustainable development of low-carbon economy in China.

Keywords: low-carbon economic development, complex network, pattern recognition, detailed spatial relationship, multi-dimensional gravity model, directed-weighted SNA

\section{Introduction}

Climate change is one of the most serious global challenges, and carbon dioxide is an important factor leading to global climate change. Therefore, reducing carbon dioxide emission has become the common goal of the international community [1]. In the Paris Climate Change Agreement launched in 2015, governments of various countries have also actively introduced relevant response policies. As an important practitioner and participant of global ecological civilization, China actively promotes the implementation of low-carbon pilot projects and the national carbon emission trading system [2, 3], and clearly puts forward the goal of reaching the peak of carbon dioxide by 2030 [4]. In this context, the low-carbon economic development pattern, aimed at reducing greenhouse gas emissions, has become one of the best choices for sustainable development in the era of climate change.

First proposed by the 2003 British Energy White Paper "The Future of Our Energy: Creating a LowCarbon Economy", the concept of low-carbon economy refers to reducing natural resource consumption and greenhouse gas emissions as much as possible, through technological innovation and institutional innovation, under the guidance of the concept of sustainable development and guided by "low energy consumption, low pollution and low emission" [5]. For a long time, China's economic growth has been characterized by "high input, high energy consumption, low efficiency and heavy pollution" [6]. As a developing country, China still aims to pursue economic growth for a long time in the future, and urgently needs to take a sustainable low-carbon economic development path [7]. However, under the concept of common development of regional integration, the low-carbon economic development of a certain province in China is promoted and restricted by the development status of itself and its surrounding provinces. It can be seen that by mining the inter-provincial detailed spatial relationship of low-carbon economy development in China, we can accurately recognize the development pattern of different provinces, make clear the development orientation, and provide some guidance and suggestions for the formulation of refined and differentiated regional development strategies, to promote the transformation of low-carbon sustainable development of China's global economy.

Since the "low-carbon economy" was proposed, the relevant research results have been fruitful. In terms of exploring the spatial correlation of lowcarbon economy development, most of the existing research, based on the evaluation of low-carbon economic development capability, mines the interregional spatial relationship by using spatial analysis tools. Among them, in the evaluation of low-carbon economic development capability, most of the existing researches construct a comprehensive evaluation index system from multi-dimensional aspects of energy, environment, economy, etc., and adopt the methods of combining subjective and objective weighting, dynamic and static methods, such as analytic hierarchy process $[8,9]$, entropy weight method [10-12], TOPSIS method [13-15], open grade method [16] and combination evaluation method [17-19], orienting to large, medium and small research scales, to comprehensively evaluate the low-carbon economic development capability of the study area. On this basis, according to the comprehensive evaluation results, some studies use spatial clustering method [20], spatial autocorrelation [15], and GIS breakpoint method [21] to analyze the spatial-temporal pattern and evolution characteristics of regional low-carbon economic development. Through combing the relevant literature, it is not difficult to find that although the research results on the evaluation of low-carbon economic development capability and its spatial-temporal distribution characteristics are relatively fruitful, the following problems still exist in the specific application process: (a) the evaluation method of low-carbon economic development capability is mainly static. When the study period is multiple annual data, it is easy to ignore 
the time series of evaluation indexes in different years, and often difficult to truly reflect the changes of lowcarbon economic development. (b) The research strength and depth of mining the detailed spatial relationship of low-carbon economic development from the perspective of relational data are insufficient. Existing research is mainly based on the attribute data of comprehensive evaluation value, and the perspective of geographical proximity, using traditional spatial econometric model, which can only roughly explore the inter-regional relationship of low-carbon economic development in geographical space. Notably, since most of the existing studies are relatively simplified and rough in mining the spatial correlation of low-carbon economic development, it is easy to make the recognition of inter-regional development patterns only stay on the simple clustering of comprehensive evaluation values. While the low-carbon economic development involves multi-dimensional elements, the inter-regional development pattern should show the characteristics of complex networked spatial relationship. Starting from multi-dimensional relational data, it is more helpful to further explore the inter-regional detailed spatial relationship, to scientifically and reasonably recognize the development patterns of different regions, and truly reflect the regional development orientation.

In recent years, Social Network Analysis (SNA) has been widely used in various fields such as sociology, economics, and geography [22-24], because it provides a new perspective based on relational data to study networked spatial relationships. Notably, although there is a lack of relevant research on the low-carbon economic development, through combing the relevant research results on economic development [25, 26], and carbon emissions [27-29], we found that, in order to facilitate network construction and analysis, most studies usually take the average value of spatial correlation strength as the threshold to obtain sparse undirected-unweighted networks through simple binarization processing. In fact, due to the influence of many factors, the inter-regional spatial relationship tends to show asymmetry, and the undirectedunweighted networks will be difficult to truly reflect the complex networked detailed spatial relationship with the characteristics of directionality and weight.

Therefore, according to the multi-dimensional elements of low-carbon economic development, and the complex networked and asymmetry of spatial relationships, this study takes 30 provinces in China as research objects, determines the spatial correlation strength of low-carbon economic development among China's provinces based on the multi-dimensional gravity model, and constructs a directed-weighted complex network. Then, from the three dimensions of overall, individual and group, we further explore the detailed spatial relationship of low-carbon economic development among China's provinces, recognize the development patterns of different provinces and clarify the development orientation, aiming to provide some guidance and suggestions for the formulation of refined and differentiated strategies to promote the coordinated and steady development of China's global low-carbon economy.

\section{Methods and Data}

Methods

\section{Complex Network Construction of China's Provincial Low-carbon Economic Development Based on Multi-Dimensional Gravity Model}

Economic development network refers to the structural form of the spatial correlation network, formed by economic subjects through various economic links such as material transportation, trade exchanges, and information transmission [30]. As a branch of economic development, low-carbon economic development involves social and ecological fields, and should present a networked spatial correlation structure. Consequently, the complex network of China's provincial low-carbon economic development in this study is an expansion of the term of economic development network. It refers to the networked spatial relationship formed by the flow of multi-dimensional elements related to the low-carbon economic development among provinces, which has the detailed spatial characteristics of directionality and weight. The key to its construction is to determine the inter-provincial spatial correlation strength of low-carbon economic development. At present, because the gravity model can better reflect the inter-regional spatial correlation, it is very promising for being used in the study of economic spatial correlation [31]. However, the existing studies mainly focus on the quality parameters represented by a single or a few indicators, and the distance parameters represented by simple geographical distance or relative geographical distance. In fact, the low-carbon economic development is influenced by multi-dimensional factors, and consequently, simplified quality and distance parameters are difficult to accurately describe the interprovincial spatial correlation. Therefore, this study improves the two parameters, and constructs a multidimensional gravity model (Formula (1)).

$$
R_{i j}=K \frac{M_{i} \times M_{j}}{D_{i j}^{b}}
$$

Where, $R_{i j}$ is the spatial correlation strength of lowcarbon economic development of China's provinces $i$ and $j ; K$ is the gravitational constant, usually taken as 1; $M_{i}$ and $M_{j}$ are the comprehensive evaluation value of low-carbon economic development capability of China's provinces $i$ and $j$, respectively; $D_{i j}$ is the comprehensive distance between China's provinces $i$ and $j$; and $b$ is the distance attenuation coefficient, which is 1.5 , based on the existing literature [32]. 
Specifically, the measurement of spatial correlation strength can be divided into two aspects: the China's provincial low-carbon economic development capability and the comprehensive distance between China's provinces.

(1) Measurement of the China's provincial low-carbon economic development capability

Considering that the low-carbon economic development involves many factors and the time series of evaluation index data, the comprehensive evaluation value of low-carbon economic development capability based on multi-dimensional evaluation index system and dynamic weight method is used to represent the quality parameters. "pressure-state-response" (PSR) model is a conceptual framework to reflect the mechanism of sustainable development, proposed by the Organization for Economic Cooperation and Development (OECD) and the United Nations Environment Programme (UNEP). It can effectively reflect the causal relationship and interaction among pressure, state and response, and is widely used in the research of sustainable development [33, 34]. Among them, "pressure" refers to the impact of human activities on regional resources and environment; "state" refers to various states of regional resources and environment under the influence of human activities; and "response" refers to the regulatory measures taken by human society to promote the sustainable development of regional social economy. Consequently, this study, based on the PSR model framework, and the connotation of low-carbon economic development, combined with the application of the index system in the representative literature of low-carbon economic development, constructs a multidimensional evaluation index system from the aspects of energy, environment, economy, society, science and technology (Table 1). The selection of indicators mainly considers the negative effects of energy consumption and pollutant discharge, as well as the positive effects of social and economic development, environmental governance and scientific and technological innovation.

In terms of the evaluation method construction, in order to ensure the time series of index data in different years during the study period, this study draws on the ungrading method proposed by Guo Yajun [35] and the standardization method of "overall standardization, time-sharing standardization" proposed by Wang Changkai [36], to construct a comprehensive evaluation model based on dynamic weight method. Specifically, (a) for the evaluation samples containing $m$ evaluation objects and $n$ evaluation indicators, according to the time sequence $t_{1}, t_{2} \ldots t_{T}$, a panel data table is constructed. (b) Combined with the direction of indicators, the panel data table is standardized (Formula (2)-(4)). (c) Based on the principle of reflecting the differences among the evaluation objects as much as possible, the index weight vector is determined (Formula (5)). (d) Based on the standardized panel data and index weights, the comprehensive evaluation value is calculated (Formula (6)).

$$
\begin{gathered}
x_{i j}^{*+}\left(t_{k}\right)=\left(x_{i j}\left(t_{k}\right)-x_{j}^{\text {min }}\right) /\left(x_{j}^{\max }-x_{j}^{\text {min }}\right), \\
i=1,2, \cdots, m ; j=1,2, \cdots, n ; k=1,2 \cdots, T \\
x_{i j}^{*-}\left(t_{k}\right)=\left(x_{j}^{\max }-x_{i j}\left(t_{k}\right)\right) /\left(x_{j}^{\max }-x_{j}^{\min }\right), \\
i=1,2, \cdots, m ; j=1,2, \cdots, n ; k=1,2 \cdots, T \\
x_{i j}^{*}=\left|x_{i j}-a\right|
\end{gathered}
$$

Where, $x_{j}^{\max }$ and $x_{j}^{\min }$ are the maximum value and the minimum value of the $j$-th index at all times, and $a$ is the appropriate center value of the center index. Among them, the positive and negative indicators are standardized by Formulas (2) and (3), respectively, while the intermediate indicators are converted into negative indicators according to Formula (4). Then, it can be processed in accordance with the standardized processing method of negative indicators.

$$
H_{k}=X_{k}^{T} X_{k}, \mathrm{H}=\sum_{k=1}^{t} H_{k}
$$

Where, $X_{k}$ is a data matrix constructed according to the standardized evaluation index value $x_{i j}{ }^{*}\left(t_{k}\right)$ in year $K . X_{k}^{T}$ is the transpose matrix corresponding to $x_{k}$. $H$ is a symmetric matrix, and the eigenvector corresponding to its maximum eigenvalue is standardized, which is the weight vector.

$$
\begin{gathered}
y_{i}\left(t_{k}\right)=\sum_{j=1}^{m} \omega_{j} x_{i j}^{*}\left(t_{k}\right), i=1,2, \cdots, m \\
j=1,2, \cdots, n ; k=1,2 \cdots, T
\end{gathered}
$$

Where, $\omega_{j}$ is the index weight; $y_{i}\left(t_{k}\right)$ is the comprehensive evaluation value of the $i$-th evaluation object at $t_{k}$.

(2) Measurement of comprehensive distance between China's provinces

Considering that the geographical proximity and the similarity of socio-economic development level will directly affect the spatial correlation strength of low-carbon economic development among China's provinces, this study uses the comprehensive value of multi-dimensional distance of geography, economy, society and adjacency to represent the distance parameter (Formula (7)). Among them, the geographical distance is calculated according to the longitude and latitude coordinates between China's provinces, the economic distance and social distance are expressed by the absolute value of the difference between China's provincial GDP per capita and the urbanization rate, and the adjacent distance is expressed by logical values 0 and 1 according to Queen's adjacent rule. In view of the attenuation of spatial correlation strength with the increase of distance, the adjacent distance between two provinces with common boundary or common vertex is regarded as 0 , otherwise it is 1 . In addition, in determining the weight of multi-dimensional distance, the weight of adjacent distance is only 0.1, 
Table 1. Multi-dimensional evaluation index system of China's provincial low carbon economic development capability.

\begin{tabular}{|c|c|c|c|c|c|}
\hline & $\begin{array}{l}\text { Primary factor } \\
\text { indexes }\end{array}$ & Secondary factor indexes & $\begin{array}{c}\text { Indexes } \\
\text { direction }\end{array}$ & Indexes description & Unit \\
\hline \multirow{7}{*}{ Pressure } & \multirow{2}{*}{$\begin{array}{l}\text { Carbon source } \\
\text { pressure }\end{array}$} & Energy consumption per unit GDP & - & \multirow{2}{*}{ Energy system } & $\begin{array}{l}\text { Tons of standard } \\
\text { coal/10,000 yuan }\end{array}$ \\
\hline & & Electricity consumption per unit GDP & - & & $\mathrm{KWh} / 10,000$ yuan \\
\hline & \multirow{5}{*}{$\begin{array}{c}\text { Pollutant } \\
\text { discharge } \\
\text { pressure }\end{array}$} & Carbon emission intensity & - & \multirow{5}{*}{$\begin{array}{l}\text { Environmental } \\
\text { system }\end{array}$} & $\begin{array}{l}\text { Ten thousand tons/one } \\
\text { hundred million yuan }\end{array}$ \\
\hline & & $\mathrm{SO}_{2}$ emissions per unit GDP & - & & Tons/100 million yuan \\
\hline & & Smoke and dust produced by unit GDP & - & & Tons/100 million yuan \\
\hline & & Output of general industrial solid waste & - & & Ten thousand tons \\
\hline & & Wastewater output per unit GDP & - & & Tons/10,000 yuan \\
\hline \multirow{16}{*}{ Condition } & \multirow{5}{*}{$\begin{array}{l}\text { Green carbon } \\
\text { sink status }\end{array}$} & Percentage of forest cover & + & \multirow{5}{*}{$\begin{array}{l}\text { Environmental } \\
\text { system }\end{array}$} & $\%$ \\
\hline & & Per capita park green area & + & & $\mathrm{m}^{2} /$ person \\
\hline & & $\begin{array}{l}\text { Coverage rate of green space in built-up } \\
\text { area } \\
\end{array}$ & + & & $\%$ \\
\hline & & Wetland area ratio & + & & $\%$ \\
\hline & & $\begin{array}{l}\text { The proportion of nature reserves in the } \\
\text { area under their jurisdiction }\end{array}$ & + & & $\%$ \\
\hline & \multirow{4}{*}{$\begin{array}{c}\text { State of } \\
\text { economic } \\
\text { development }\end{array}$} & $\begin{array}{l}\text { Total exports of domestic destinations and } \\
\text { sources of goods }\end{array}$ & + & \multirow{6}{*}{ Economic system } & Millions of dollars \\
\hline & & Gross regional product (GDP) & + & & $\begin{array}{l}\text { One hundred million } \\
\text { yuan }\end{array}$ \\
\hline & & Per capita GDP & + & & Yuan/person \\
\hline & & $\begin{array}{l}\text { Investment in fixed assets of the whole } \\
\text { society }\end{array}$ & + & & $\begin{array}{l}\text { One hundred million } \\
\text { yuan }\end{array}$ \\
\hline & \multirow{2}{*}{$\begin{array}{c}\text { State of } \\
\text { industrial } \\
\text { structure }\end{array}$} & Proportion of secondary industry & + & & $\%$ \\
\hline & & The tertiary industry accounted for & + & & $\%$ \\
\hline & \multirow{3}{*}{$\begin{array}{l}\text { Residents' } \\
\text { living } \\
\text { conditions }\end{array}$} & Urban per capita disposable income & + & \multirow{5}{*}{ Social system } & Yuan \\
\hline & & Consumption level of urban residents & + & & Yuan \\
\hline & & Consumption level of rural residents & + & & Yuan \\
\hline & $\begin{array}{l}\text { Traffic trip } \\
\text { status }\end{array}$ & $\begin{array}{c}\text { Public transport vehicles per } 10,000 \\
\text { people }\end{array}$ & + & & $\begin{array}{l}\text { Vehicles } / 10,000 \\
\text { people }\end{array}$ \\
\hline & $\begin{array}{c}\text { State of social } \\
\text { development }\end{array}$ & Urbanization rate & $\begin{array}{c}\text { Centered } \\
\text { type }\end{array}$ & & $\%$ \\
\hline \multirow{7}{*}{ Respond } & \multirow{5}{*}{$\begin{array}{l}\text { Pollution } \\
\text { control }\end{array}$} & $\begin{array}{c}\text { Comprehensive utilization of general } \\
\text { industrial solid waste }\end{array}$ & + & \multirow{5}{*}{$\begin{array}{l}\text { Environmental } \\
\text { system }\end{array}$} & Ten thousand tons \\
\hline & & Industrial wastewater treatment capability & + & & Ten thousand tons \\
\hline & & $\begin{array}{l}\text { Treatment capability of industrial waste } \\
\text { gas treatment facilities }\end{array}$ & + & & $\begin{array}{l}\text { Ten thousand cubic } \\
\text { meters/hour }\end{array}$ \\
\hline & & $\begin{array}{l}\text { Harmless treatment rate of domestic } \\
\text { garbage }\end{array}$ & + & & $\%$ \\
\hline & & Urban sewage treatment rate & + & & $\%$ \\
\hline & \multirow{2}{*}{$\begin{array}{l}\text { Scientific and } \\
\text { technological } \\
\text { level }\end{array}$} & Investment intensity of R\&D funds & + & \multirow{2}{*}{$\begin{array}{l}\text { Science and } \\
\text { technology system }\end{array}$} & $\%$ \\
\hline & & $\begin{array}{c}\text { Authorized amount of domestic patent } \\
\text { application }\end{array}$ & + & & Item \\
\hline
\end{tabular}


mainly because it is used to highlight the spatial correlation strength between two provinces with adjacent relationship, and can avoid excessively limiting the correlation relationship to the adjacent effect. Finally, the comprehensive distance is prescribed to match the order of magnitude of the comprehensive evaluation value of provincial low-carbon economic development capability.

$$
D_{i j}=\sqrt{\omega_{1} \times\left(D_{1}+D_{2}+D_{3}\right)+\omega_{2} \times D_{4}}
$$

Where, $\omega_{1}$ is the weight of geographical distance $D_{1}$, economic distance $D_{2}$ and social distance $D_{3} ; \omega_{2}$ is the weight of adjacent distance $D_{4}$. After several calculations and adjustments, the final values are 0.3 and 0.1 , respectively.

In order to truly reflect the inter-provincial complex networked detailed spatial relationship with directed and weighted characteristics in the lowcarbon economic development in China, this study constructs a directed-weighted complex network according to the measurement results of inter-provincial spatial correlation strength matrix. It is ensured that the research on the pattern recognition of China's provincial low-carbon economic development carried out on this basis is scientific and reasonable.

\section{Recognition of China's Provincial Low-Carbon Economic Development Pattern Based on the Detailed Spatial Relationship}

In order to deeply explore the low-carbon economic development patterns of different provinces in China, this study, based on the directed-weighted complex network of China's provincial low-carbon economic development, explores the inter-provincial detailed spatial relationship of low-carbon economic development in China from three dimensions of overall, individual and group. It aims to further identify the specific development pattern and its evolution trend of each province and clarify the development orientation, to provide some guidance and suggestions for the formulation of refined and differentiated regional low-carbon economic development strategies. Among them, the overall dimension mainly refers to the recognition of the inter-provincial correlation structure of low-carbon economic development, the individual dimension mainly refers to the recognition of network centers, and the group dimension mainly refers to the recognition of cohesive subgroups.

(1) Correlation structure

Based on the network density and spatial correlation strength, this study identifies the correlation structure of inter-provincial low-carbon economic development in China. Among them, network density is used to measure the inter-provincial closeness relationship of low-carbon economic development in the network as a whole. Considering that the density of directedweighted complex network is affected by both the number and strength of correlation lines, an improved calculation method is proposed in this study, based on the previous calculation methods for undirected-unweighted network (Formula (8)).

$$
D_{n}=\sum_{i=1}^{n} \sum_{j=1}^{n} R_{i j} /[n(n-1)]
$$

Where, $D_{n}$ is the network density of province $n ; n$ is the number of provinces; and $R_{i j}$ is the spatial correlation strength processed by "overall standardization and timesharing standardization" to ensure the comparability of data in different years.

(2) Network center

Network center is used to measure the importance of the location of a certain province in the network, which is usually determined according to the degree centrality. For the directed-weighted complex network, the degree centrality of a province is related to the number of provinces with association relationship, and the direction and weight of association relationship [37]. The specific calculation method is shown in Formula (9).

$$
\begin{gathered}
\mathrm{S}(i)=k_{i} \times\left(s_{i} / k_{i}\right)^{\alpha}, \mathrm{C}(i) \\
=\sqrt[3]{S_{\text {out }}(i) \times S_{\text {in }}(i) \times \frac{1}{2}\left(S_{\text {out }}(i)+S_{\text {in }}(i)\right)}
\end{gathered}
$$

Where, $S(i)$ is the spatial correlation strength of province $i$, which is divided into outgoing strength $S_{\text {out }}(i)$ and incoming strength $S_{i n}(i) ; S_{i}$ is the weight sum of all edges connected with province $i$; $k_{i}$ is the number of provinces adjacent to province $i ; C(i)$ is the centrality of province $i$ in the network. Considering that the value of $\alpha$ has almost negligible influence on the calculation result of the centrality of the directed-weighted complex network, it is set to 1 for the convenience of calculation.

(3) Cohesive subgroups

Cohesive subgroups are based on the relatively strong, direct, close, positive or frequent relationship between different provinces in subgroup, and divide the network into different small groups, to grasp the similarity and difference of the inter-provincial relationship from the group scale. In view of this, this study uses the Convergent Correlation method (CONCORE), which emphasizes the correlation relationship, to analyze the cohesive subgroups of the complex network of China's provincial low-carbon economic development.

\section{Study Area and Data Sources}

This study takes China's provincial administrative regions as the research object (collectively referred to as "China's provinces"). Due to the serious lack of data in some provinces in the statistical yearbook, it is difficult to predict and supplement with statistical methods. Therefore, Tibet, Hong Kong, Macao and Taiwan are excluded, totaling 30 provinces. China has 




Fig.1. Regional division map of 30 provinces in China.

a vast territory, and the provinces are uneven in industrial structure, resource endowment and socioeconomic development level. The low-carbon economy has also developed under this unbalanced state. This study, taking 30 provinces as the research object, can include the relevant provinces of the four regional development strategic patterns, which includes first development of the eastern region, the rise of the central region, the development of the western region, and the revitalization of the northeast region (Fig. 1). In addition, in order to truly reflect the relatively stable low-carbon economic development patterns presented by China's provinces in a long time series as much as possible, and avoid the serious lack of index data in individual provinces in individual years, this study takes the 10 years from 2008 to 2017 as the research period. It can ensure that the research can be carried out within a relatively long time series and with relatively complete index data, ensure the scientificity and rationality of the research results, and help to guide the formulation of refinement and differentiation development strategies.

The original data used in this study mainly include energy consumption, social economy, environmental resources, scientific research patents and geographic data. The relevant statistical data are mainly from China Energy Statistical Yearbook, China Statistical Yearbook, China Environmental Statistical Yearbook, and the scientific and technological statistical analysis report of the Ministry of Science and Technology of the People's Republic of China from 2009 to 2018. The administrative division data comes from the National Geographic Information Center.

\section{Results and Discussion}

Comprehensive Analysis of China's Provincial Low-Carbon Economic Development Capability with Long Time Series

According to the multi-dimensional evaluation index system and dynamic weight evaluation model constructed above, on the basis of ensuring the statistical caliber consistency and standardized processing of index data, the low-carbon economic development capability in 30 provinces in China from 2008 to 2017 is comprehensively measured, to ensure the comparability of measurement results in different years.

Fig. 2 presents the growth trend of low-carbon economic development capability of all provinces in China with different rates of increase. From the research year, the low-carbon economic development capability of different provinces is quite different. In the past 10 years, Jiangsu, Zhejiang, Guangdong, Beijing, Fujian, and Shandong have maintained

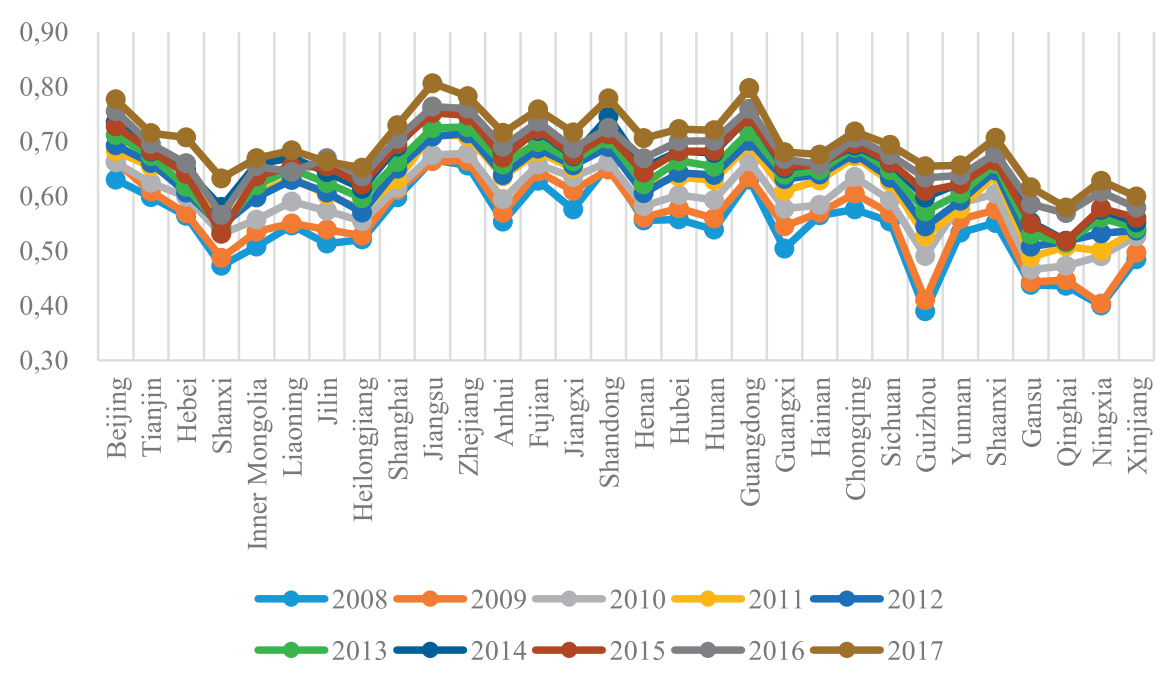

Fig. 2. Comprehensive evaluation value of China's provincial low-carbon economic development capability (2008-2017). 
a superior level of low-carbon economic development; Shanghai, Jiangxi, Tianjin, Chongqing, and Hubei have maintained a good level; An-hui, Shaanxi, Hunan, and Sichuan have maintained an upper-middle-level with a good development situation; while Shanxi, Guizhou, Gansu, Qinghai, Ningxia, and Xinjiang have remained at a low-level. In low-carbon economic development, the provinces located in the eastern coastal areas have the best development capability, followed by the central area, and the western area is worse. This is consistent with the research results and trend of literature [13, 38], indicating that the evaluation results of this study are reasonable. In general, during the study period, China's provincial low-carbon economic development capability has been continuously improved, but it has shown an unbalanced development situation. The overall situation is from strong to weak, from east to west, and there is a jump pattern in some areas.

\section{Pattern Recognition and Evolution of China's Provincial Low-Carbon Economic Development with Long Time Series}

\section{Recognition of Correlation Structure}

According to the multi-dimensional gravity model, the inter-provincial spatial correlation strength of low-carbon economic development is determined, and the directed-weighted complex network is constructed. From Fig. 3, the network density of China's provincial low-carbon economic development complex network is increasing year by year, from 0.26 in 2008 to 0.41 in 2017, indicating that China's provincial low-carbon economic development is increasingly closely related.

Considering the small change in network density during consecutive years during the study period, indicating the correlation structure has not changed much in consecutive years, and limited by space, taking three years as the interval, this study selects four time nodes to further analyze the spatial correlation strength and the change of correlation structure of China's provincial low-carbon economic development.

From Fig. 4, from the change of spatial correlation strength, during the study period, the number of

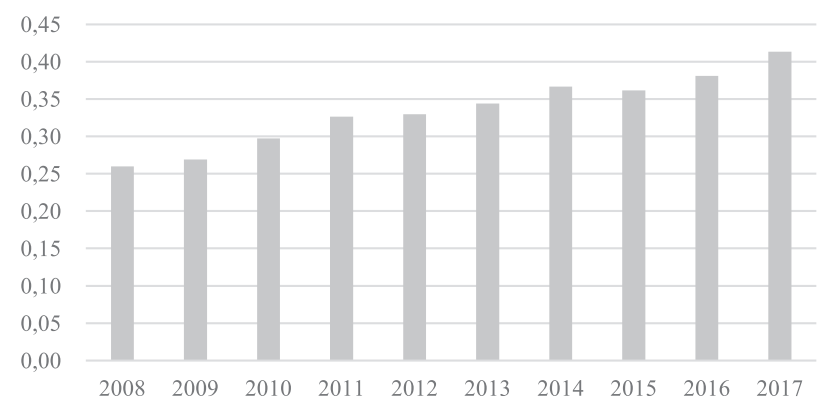

Fig. 3. The network density of China's provincial low-carbon economic development complex network (2008-2017). provincial pairs with spatial correlation strength greater than 5 gradually increased, especially the provincial pairs in the interval of $(5,10)$ and $(10,20)$. By 2017 , the number of provincial pairs with spatial correlation strength less than 5 has decreased considerably. Among them, provincial pairs with spatial correlation strength greater than 20 mainly exist between a few surrounding provinces, such as Beijing-Tianjin, Jiangsu-Zhejiang, and Yunnan-Guizhou, while the provincial pairs located in the $(10,20)$ interval are mainly concentrated in the central and eastern coastal regions.

Considering that the low-carbon economic development in each province has a few provinces which are closely related to it, and the characteristics of correlation structure are mainly determined by the provincial pairs with close relationship, this study selects the maximum and the top five spatial correlation strength of each province for further exploring the detailed spatial relationship characteristics of correlation structure.

From Fig. 5, it can be found that: (a) the interprovincial spatial relationship is obviously limited by geographical spatial factors, and geographically adjacent provinces are more likely to show close spatial relationship with each other, such as BeijingTianjin, Jilin-Heilongjiang, and Jiangsu-Zhejiang. (b) The inter-provincial spatial relationship is asymmetric. For example, in 2008, Jiangsu and Hunan were the provinces with the closest spatial relationship with each other. By 2011, Hunan and Jiangsu had the closest spatial relationship, but Jiangsu and Anhui showed the closest spatial relationship. It can be seen that the spatial interaction between Hunan and Jiangsu is asymmetric. (c) Although the closest spatial relationship between provinces shows some differences in different years, it remains relatively stable on the whole.

From Fig. 6, it can be found that: (a) the number of spatial correlation lines of provincial pairs gradually increases, and the complexity of spatial relationship is deepening, showing a multi-linear and complex network correlation structure. (b) The geographical proximity and asymmetry of inter-provincial spatial relationship are further highlighted. (c) The provinces in the central and eastern regions such as Jiangsu, Zhejiang, Jiangxi, Shandong, Hubei, Hunan, and Shaanxi can not only send strong relationships to other provinces, but also receive strong relationships from other provinces.

It can be seen that the inter-provincial spatial correlation of low-carbon economic development is increasingly close, showing a complex networked correlation structure, which is characterized by multi-linearity, complexity, asymmetry, and geographical proximity. This is mainly due to the continuous improvement of the overall low-carbon economic development capability and the promotion of the regional socio-economic coordinated and integrated development strategy, but also reflects the imbalance of regional spatial correlation. Among them, the provinces located in the central and eastern 


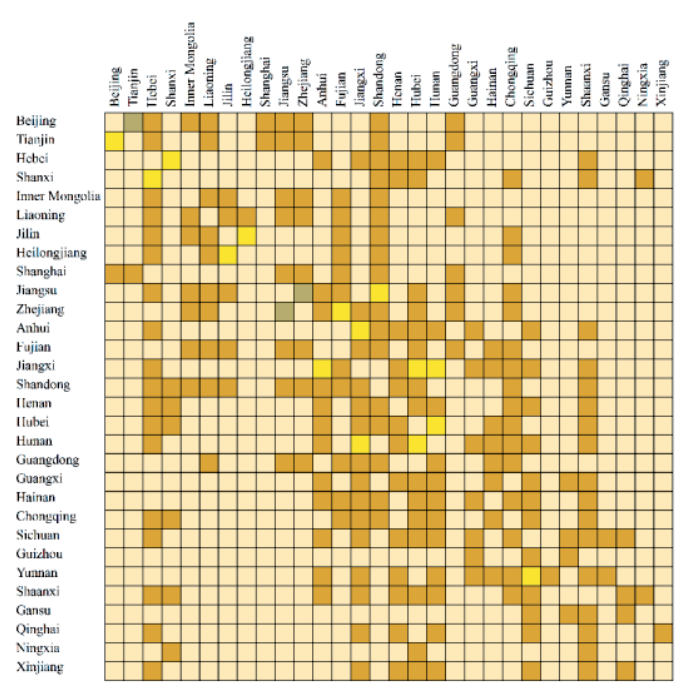

(a) 2008

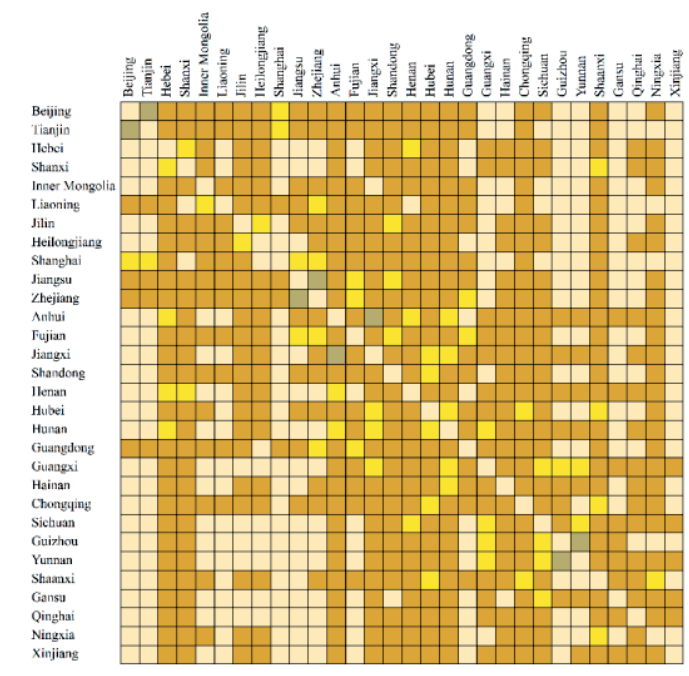

(c) 2014

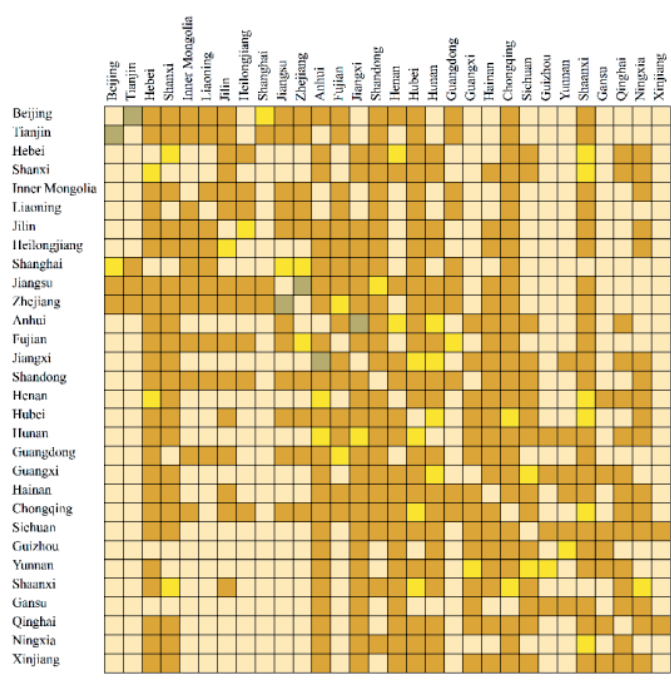

(b) 2011

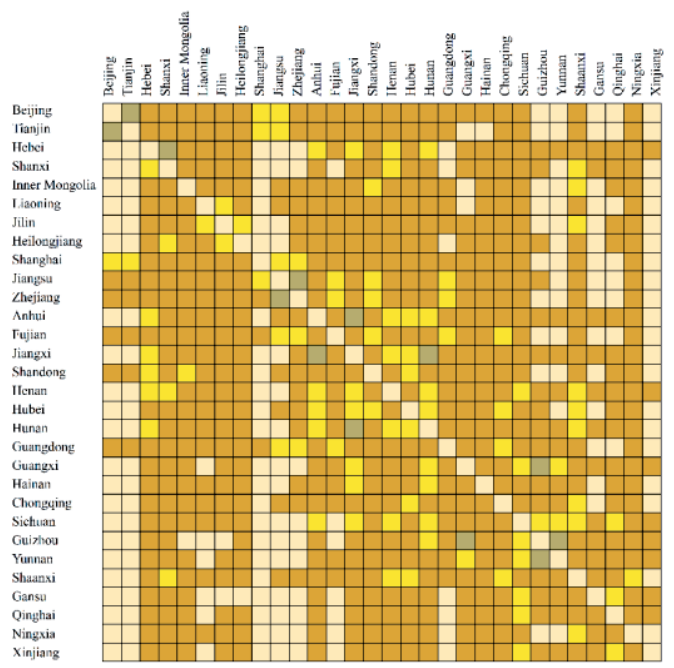

(d) 2017

Spatial correlation strength $>20$ $(10,20]$ $(5,10] \square \leq 5$

Fig. 4. The spatial correlation strength of China's provincial low-carbon economic development (2008, 2011, 2014, 2017).

coastal regions of China are more likely to have a closer spatial relationship with other provinces, which facilitates the flow and sharing of resource elements, thereby promoting mutual low-carbon economic development. It can be seen that the low-carbon economic development in a province is no longer an independent individual, but is easily affected by the promotion and restriction of surrounding provinces.

In general, the complex network of China's provincial low-carbon economic development can be roughly divided into five regions: the eastern coastal areas including Beijing and Tianjin, centered on Hubei, Hunan and Jiangxi provinces in Central China and including the surrounding provinces, the northeast region, the northwest region and the southwest region. This network structure is consistent with the strategic pattern of China's four regional economic development. On this basis, it is scientific and reasonable to recognize the network center and cohesive subgroups.

\section{Recognition of Network Center}

According to Formula (9), the degree centrality of each province in the complex network of low-carbon economic development during the study period is calculated and sorted in descending order according to the numerical value, as shown in Fig. 7.

It can be found that, (a) from the perspective of the provincial members of each interval, during the study period, except for individual provinces such as Chongqing, Guizhou, and Ningxia, the provincial members ranking within [0-5] did not change greatly, that is to say, the centrality ranking of the provinces in the complex network of low-carbon economic development is relatively stable, indicating that the importance of the provinces' positions in the network has not changed significantly, showing a certain degree of solidification. (b) Jiangxi, Hubei, Hunan, Shaanxi, and Anhui have always been among the best, indicating 


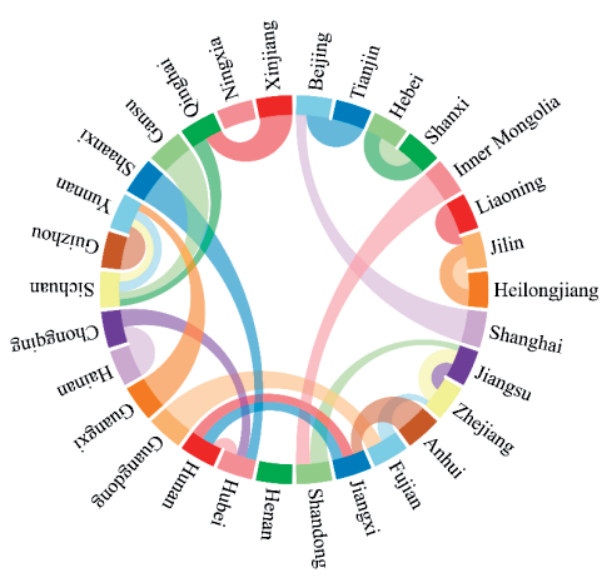

(a) 2008

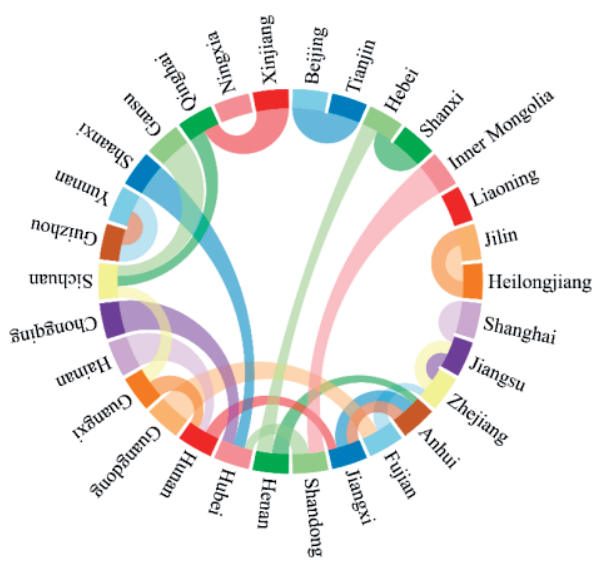

(c) 2014

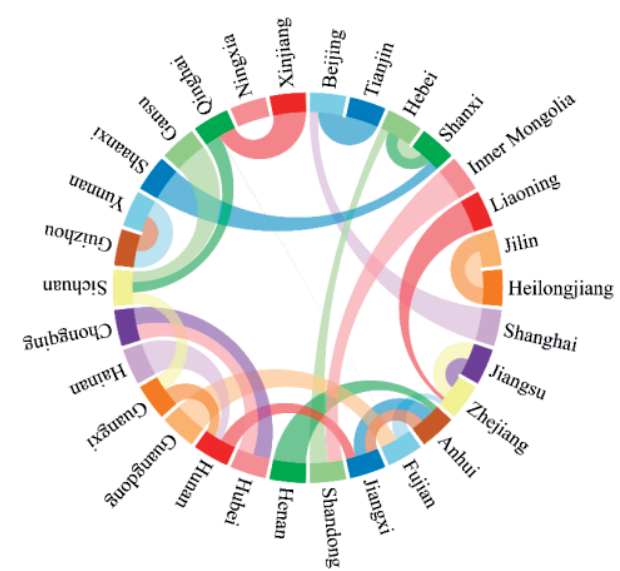

(b) 2011

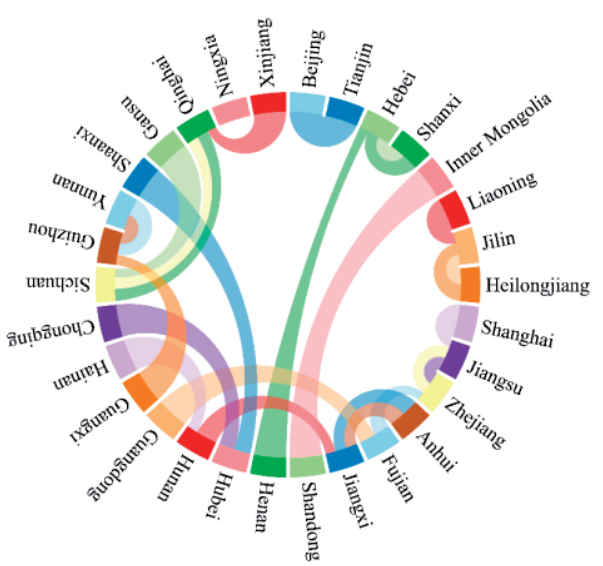

(d) 2017

Fig. 5. Correlation structure of China's provincial low-carbon economic development complex network (TOP1) (2008, 2011, 2014, 2017). Note: The thickness of the connection represents the size of the spatial correlation strength, and the corresponding circle in the province represents the sum of the spatial correlation strength. Fig. 6 is the same as.

that they are in the core position of the network and belong to the network center. Hebei, Shanxi, Chongqing, Sichuan, Shandong, Jiangsu, and Zhejiang are basically stable in the middle and upper levels, indicating that they are in a more important position in the network, second only to the network center, and are easy to have spatial correlation with the surrounding provinces. Qinghai, Gansu, Xinjiang, and Heilongjiang are always at the back end of the ranking, indicating that they are at the edge of the network. (c) Notably, the centrality of Beijing, Tianjin, Shanghai, and Guangdong with obvious advantages in low-carbon economic development and socio-economic development capability is not high. This is mainly because the measurement of centrality of this study is based on the total outgoing and incoming degrees of spatial correlation strength. Among them, the outgoing degree represents the radiation effect caused by sending spatial correlation to other provinces, and the incoming degree represents the reception effect caused by receiving spatial correlation of other provinces. Specifically, taking Beijing as an example, it is easy for Beijing to produce strong radiation effects to the surrounding provinces, especially those with the same development capability as Tianjin and Shanghai. However, in addition to generating strong reception effects with them, the reception effects with other provinces are weak, resulting in lower incoming degree and lower centrality. (d) On the contrary, Hebei, whose low-carbon economic development and socio-economic development capability are both on the middle level, has a higher degree of centrality. Specifically, Hebei is easy to produce radiation and reception effects with multiple other provinces at the same time. Although the spatial correlation strength between provinces with strong correlation relationship is not as strong as Beijing, it can basically be maintained at the average value and above. In this way, combining the spatial correlation strength with multiple provinces, the total value of incoming degree and outgoing degree is not low, and then shows a high centrality. The above phenomenon is mainly attributed to the fact that the measurement of comprehensive distance in this study integrates the economic and social distance, measured based on per capita GDP and urbanization rate. Specifically, Beijing's per capita GDP and urbanization rate far exceed most provinces in China, resulting in a large comprehensive 


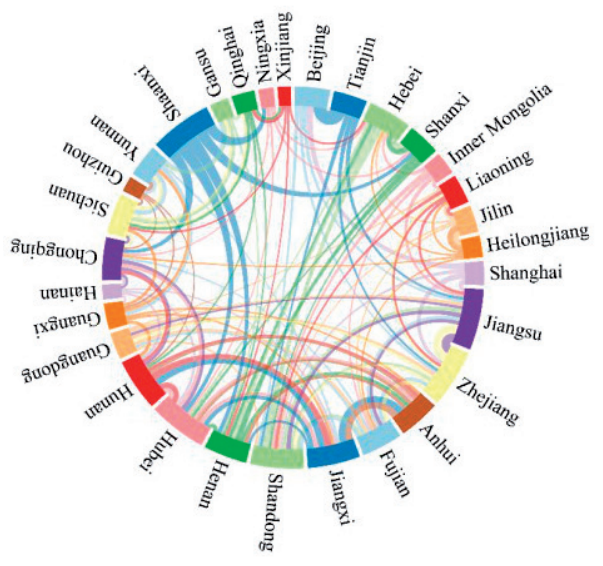

(a) 2008

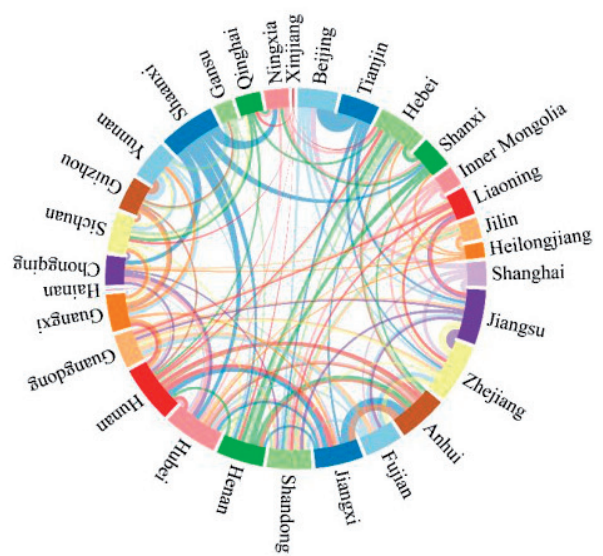

(c) 2014

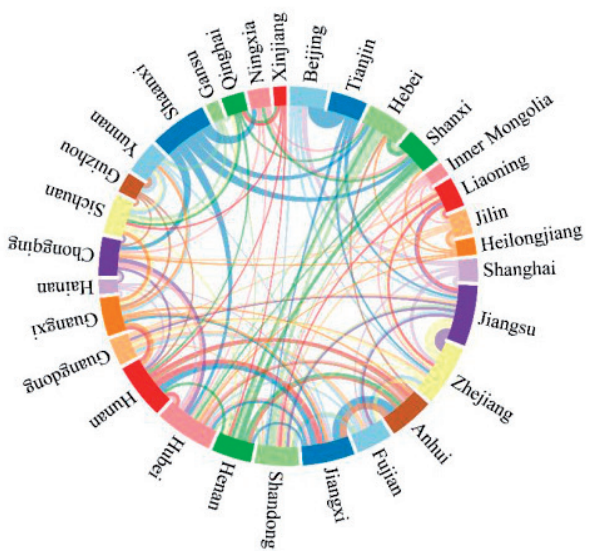

(b) 2011

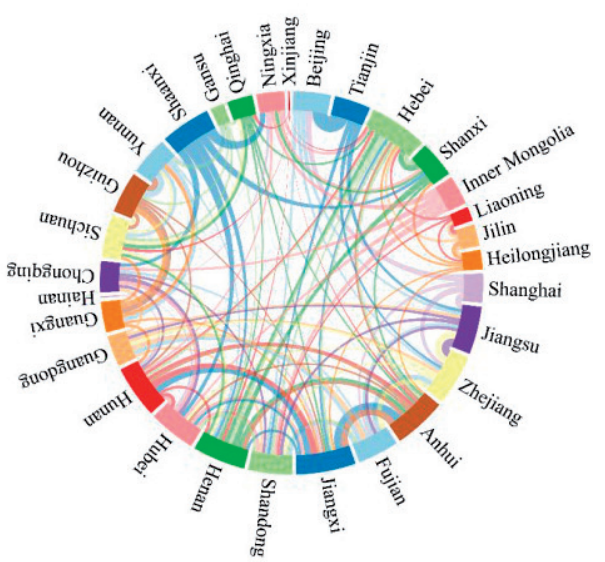

(d) 2017

Fig. 6. Correlation structure of China's provincial low-carbon economic development complex network (TOP5) (2008, 2011, 2014, 2017).

distance from most provinces, which makes it difficult to accept the strong spatial correlation sent by these provinces. However, Hebei is located in the connecting position between the eastern coastal area and the central area. Concurrently, the medium development capability makes it easy to have a spatial correlation with most provinces with high and low development capability. It can be seen that, the importance of a province's position in the low-carbon economic development complex network depends not only on its own development

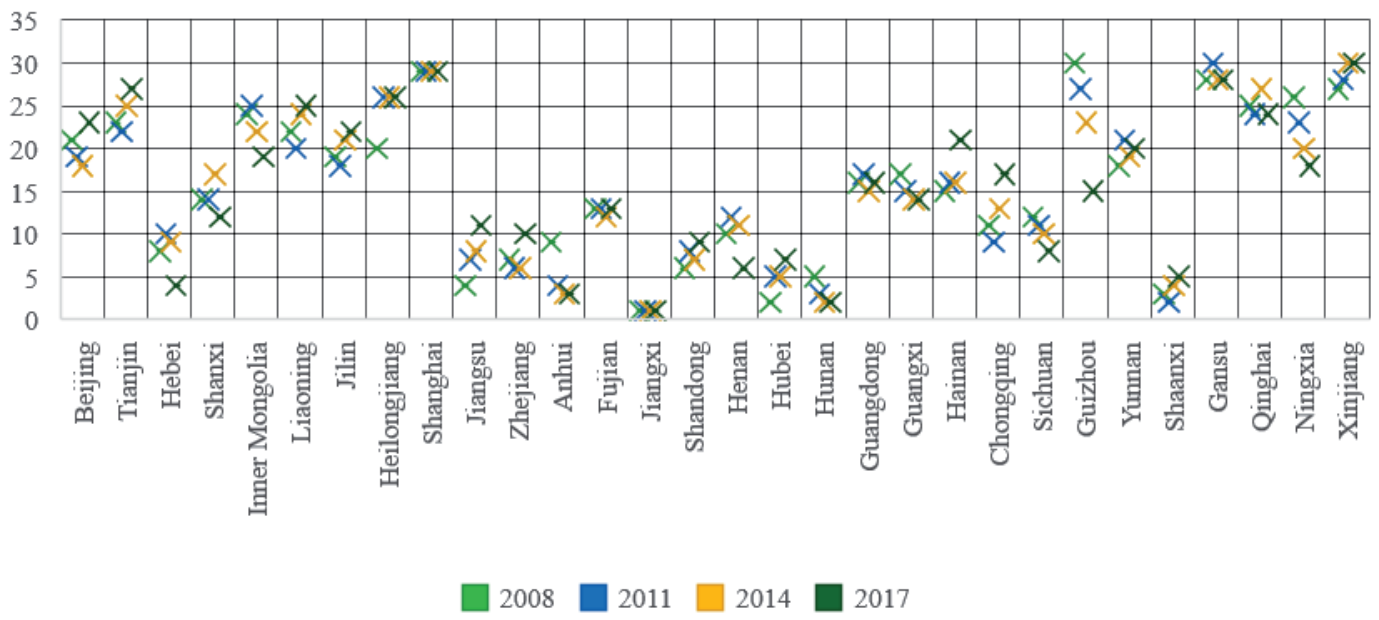

Fig. 7. The centrality ranking of China's provincial low-carbon economic development complex network $(2008,2011,2014,2017)$. 
capability, but also on its geographical advantage of connecting the east and the west and, concurrently, that the development capability gap with neighboring provinces is not too large.

It can be seen that although geographically adjacent provinces are easy to have strong correlation with each other, if the development capability gap between them is too large, the spatial correlation strength will be weakened to a certain extent. At this time, if the interprovincial spatial correlation is considered only from the perspective of geospatial proximity, it is often easy to strengthen the spatial correlation between adjacent provinces. Concurrently, it is easy for the strong to send a strong correlation to the weak to exert the radiation effect; however, because of the limited development capability of the weak, it is generally difficult to produce the same radiation effect on the strong. However, in this process, provinces with medium and above medium capability can undertake to transfer the radiation effect of the strong to the weak, showing the hierarchy of radiation effect, further highlighting their importance in promoting the development capability of China's global low-carbon economy. This is consistent with the law of regional development, and can further verify the rationality of this study in the measurement of interprovincial spatial correlation strength and centrality.

\section{Recognition of Cohesive Subgroups}

From Fig. 8, according to the cohesive subgroups' results of the complex network of China's provincial low-carbon economic development, it can be found that, (a) from the number of subgroups, the complex network of China's provincial low-carbon economic development has formed eight subgroups in each year. Except that individual provinces and non-adjacent provinces can be condensed into the same subgroup, for example, Shandong and Chongqing were condensed into subgroup in 2017, basically the provinces covered by the same subgroup are adjacent to each other in geographical space. Visible, the spatial correlation structure of low-carbon economic development between geographically adjacent provinces is more similar, which is conducive to the flow of resource elements between them, but at the same time it is easy to limit the radiation scope and strength of low-

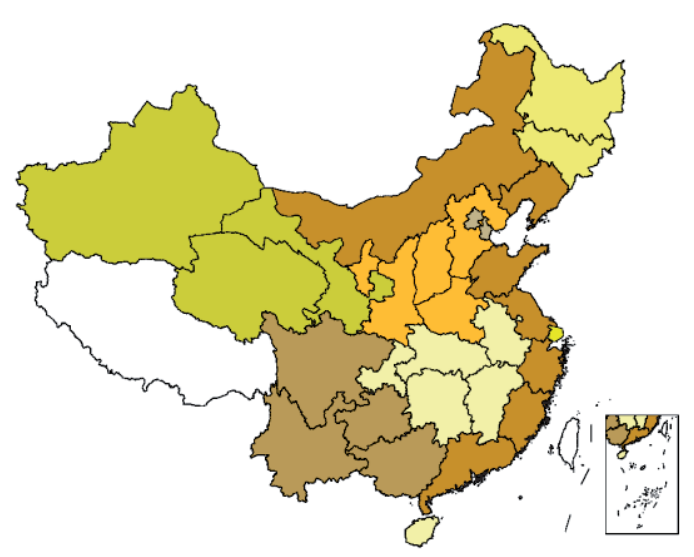

(a) 2008

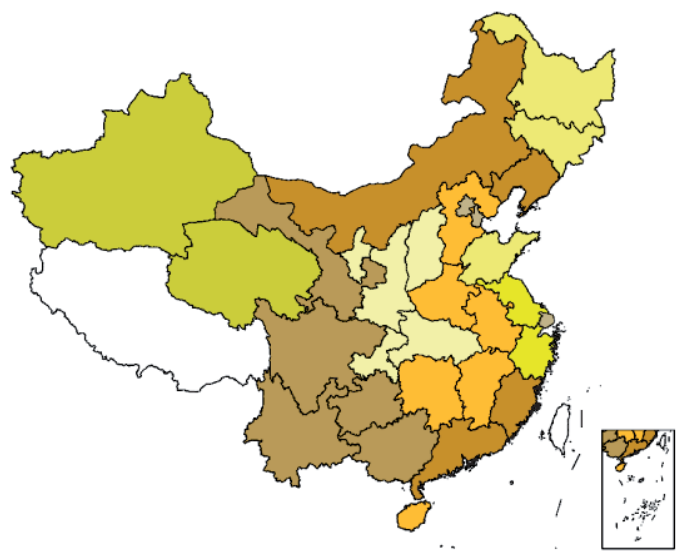

(c) 2014
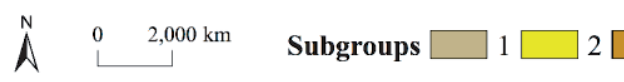

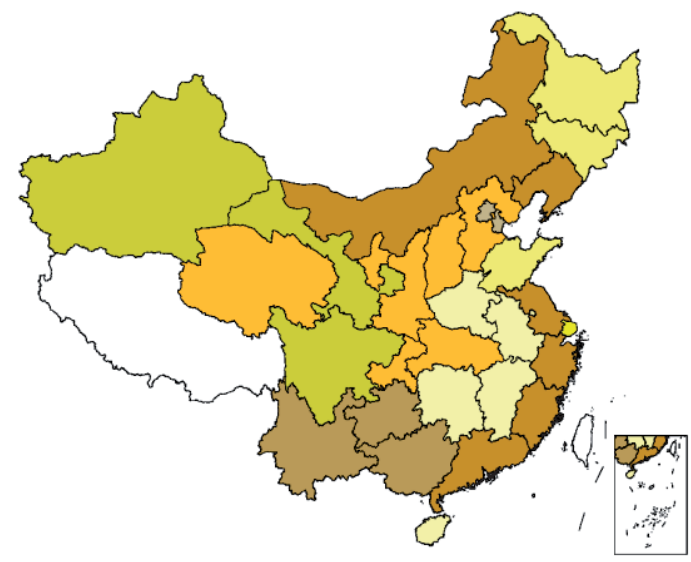

(b) 2011

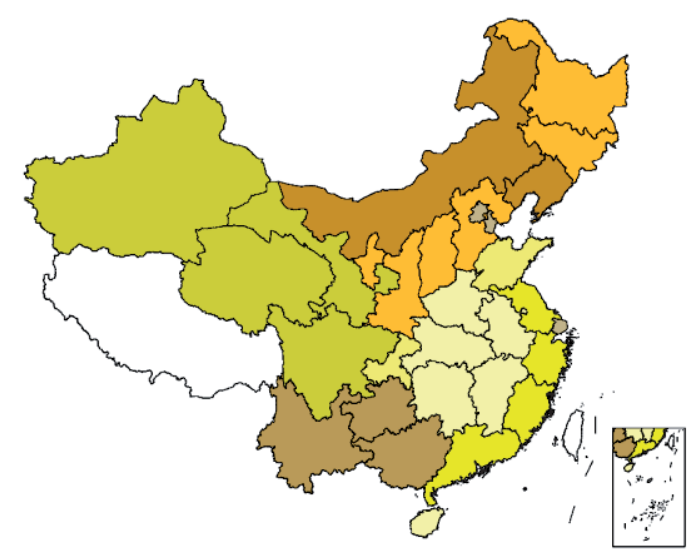

(d) 2017



Fig. 8. Cohesive subgroup distribution of China's provincial low-carbon economic development complex network (2008, 2011, 2014, 2017). Note: The same color represents the same subgroup 
carbon economic development in peripheral provinces. (b) From the changes of the provincial members of each subgroup, although the number of subgroups is the same in different years, the provinces included in each subgroup are not static. For example, in 2008 and 2011, Guangdong, Fujian, Zhejiang, Jiangsu, Liaoning and Inner Mongolia condensed into the same subgroup. In 2014, Jiangsu and Zhejiang were separated from it and condensed into subgroup, while the remaining provinces were condensed into another subgroup. By 2017, Guangdong, Fujian, Zhejiang and Jiangsu are condensed into a subgroup, while Liaoning and Inner Mongolia are condensed into another subgroup. Visible, the cohesive process of the subgroups presents a constantly changing characteristic, which is mainly due to the competition between different provinces brought about by the continuous development and changes of the social and economic development and the low-carbon economic development capability of different provinces. However, it's not difficult to find that, even though the process of cohesion between different provinces in the network evolves constantly, the overall cohesive direction of the network has not changed greatly, and finally it is stable to cluster among provinces with similar development level and geographical proximity.

\section{Pattern Recognition and Its Evolution}

By superimposing the measurement result of the comprehensive evaluation value of China's provincial low-carbon economic development capability and centrality, it is divided into four grades according to the natural breakpoint method. Fig. 9 shows that the unbalanced development situation of China's provincial low-carbon economic development capability. Specifically, the provinces in the eastern coastal areas have superior development capability, and their neighboring provinces in the central region are at the network center. They have always maintained the excellent development of low-carbon economy, which is the result of multi center drive. Concurrently, it is easy to form a virtuous cycle of "promoting development" among each other. However, the northeast, northwest and some southwest provinces are at the edge of the network and lack of self-development power,

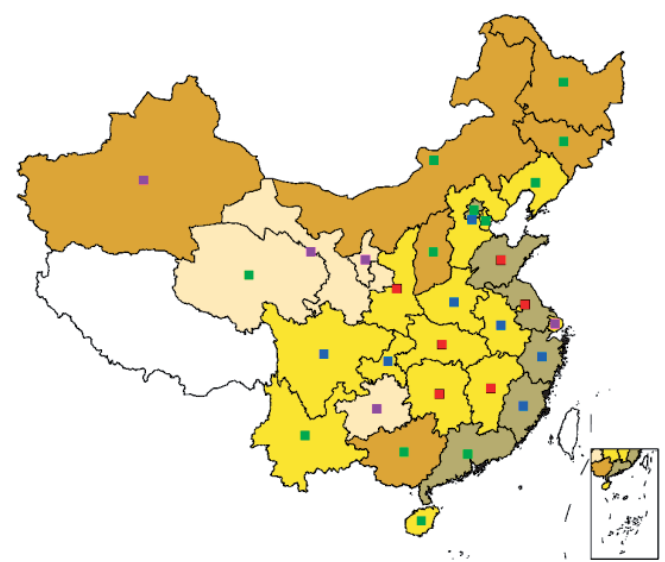

(a) 2008

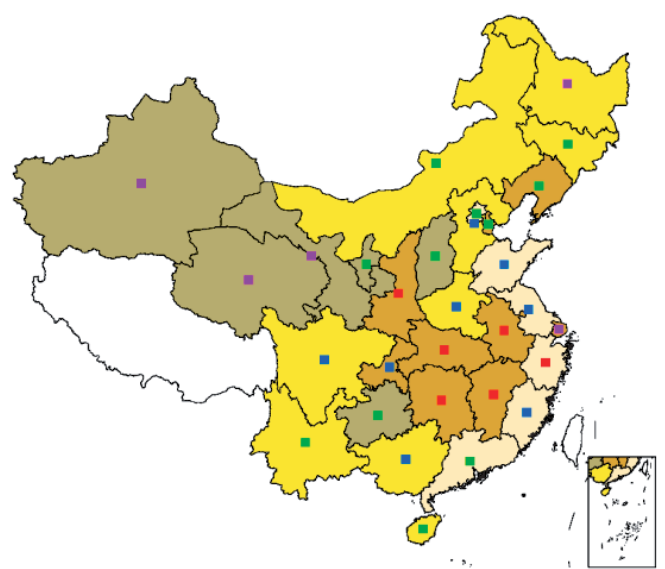

(c) 2014



(b) 2011



(d) 2017



Fig. 9. Low-carbon economic development capability and centrality level distribution (2008, 2011, 2014, 2017). Note: the numerical value is $\mathrm{I}>\mathrm{II}>\mathrm{II}>\mathrm{IV}$. 
so it has been difficult to improve the development capability of low-carbon economy.

The low-carbon economic development of a certain province is not only affected by its own development status, but also interacts with neighboring provinces through correlation and interaction effects. At this time, it is not comprehensive enough to identify the development patterns only based on the comprehensive evaluation value of the low-carbon economic development capability. Concurrently, the previous research results in the spatial correlation structure and centrality are consistent with the regional economic development strategy and development law. Considering this, it is necessary to deeply explore the development patterns of China's provincial low-carbon economy from the perspective of networked spatial correlation, clarify the development orientation of each province, to provide guidance for the formulation of fine and differentiated development strategies, and then promote the coordinated development of China's global lowcarbon economy.

According to the comprehensive analysis results of low-carbon economic development capability, centrality and cohesive subgroups, and considering the differences in geographical and spatial location, China's low-carbon economic development pattern during the study period is divided into four types: spillover, main bridge, auxiliary bridge, and benefit type. Specifically, (a) The spillover type mainly refers to provinces with excellent low-carbon economic development capability and low centrality. They are easy to send strong correlation to the surrounding provinces, and radiate and drive the low-carbon economic development in the surrounding provinces by giving play to the spatial spillover effect. (b) The main bridge type mainly refers to the provinces with excellent low-carbon economic development capability and high centrality. They are located in the center of the complex network, and are easy to have a two-way correlation between radiation and reception with most surrounding provinces. Concurrently, they are generally close to spillover provinces and have a small gap with these provinces, which is more convenient to receive the radiation effect of these provinces. On this basis, by superimposing its own development advantages, it further radiates and drives the low-carbon economic development in adjacent provinces, to play the role of bridge support. (c) The auxiliary bridge type mainly refers to provinces

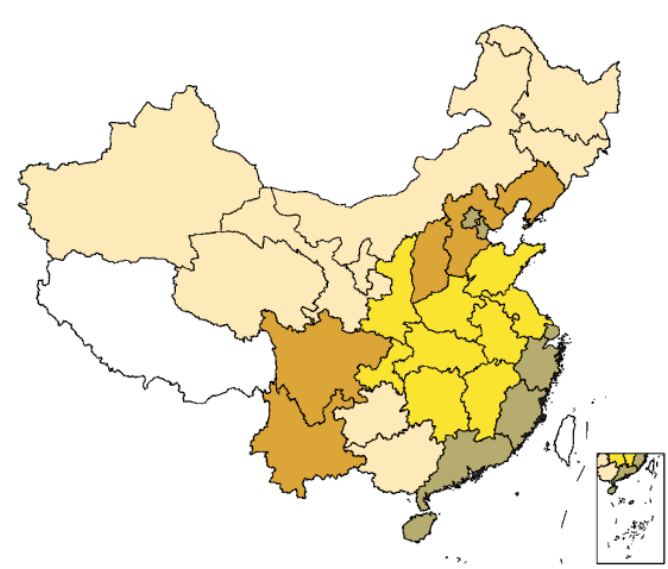

(a) 2008

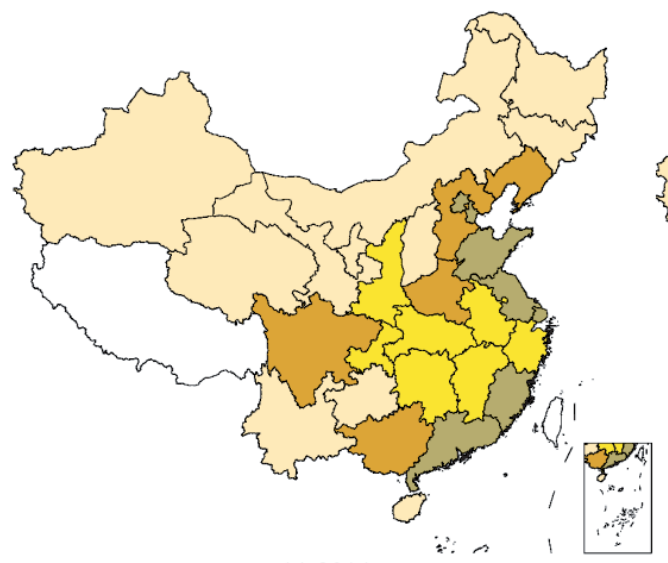

(c) 2014

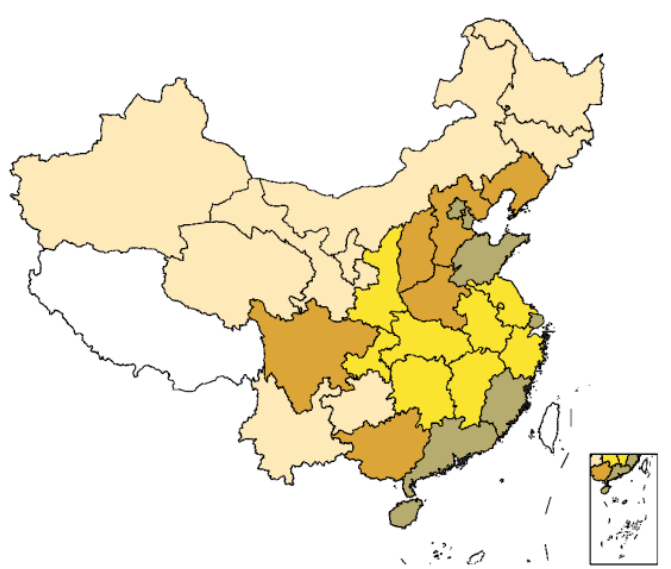

(b) 2011

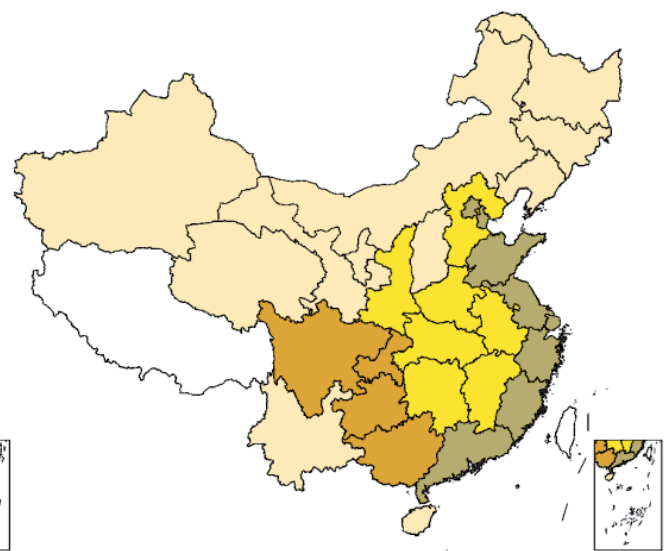

(d) 2017

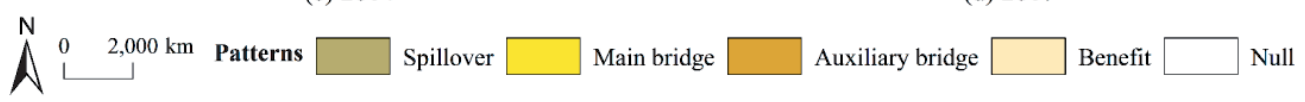

Fig. 10. Recognition results of China's provincial low-carbon economic development pattern $(2008,2011,2014,2017)$. 
with medium low-carbon economic development capability and high centrality. They are generally adjacent to spillover and main bridge provinces, and are easy to receive the radiation effects from these provinces, especially the main bridge provinces with small development capability gap. Concurrently, due to the medium development capability, it is easy to maintain a similar development pattern with the provinces with poor development capability, and then have a strong spatial correlation with these provinces. However, due to the medium development capability and limited radiation driving effect, it can only undertake the auxiliary bridge type to drive the development of the surrounding provinces. (d) The beneficial type mainly refers to provinces where the low-carbon economic development capability and centrality are at a disadvantage. Most are located at the edge of the network, and their own development power is insufficient; therefore, they urgently need to rely on the radiation and driving role of other provinces.

Based on the above four development patterns, the China's provincial low-carbon economic development patterns during the study period are divided. Fig. 10 shows that there are small changes in the provincial members included in each development pattern. For example, Jiangsu belonged to the main bridge type in 2008 and 2011, while it belonged to the spillover type in 2014 and 2017; Guangxi belonged to the benefit type in 2008, while other years belonged to the auxiliary bridge type. The reason is that due to the differences in the layout of development strategies, it is easy for provinces to form a competitive situation in the lowcarbon economic development capability and centrality, which is finally reflected in the changes of development pattern. However, in general, during the study period, the development pattern of China's provincial lowcarbon economy has not changed greatly. Concurrently, the above result of cohesive subgroups also show that the development pattern of China's provincial lowcarbon economy has a relatively stable state. Therefore, a basically stable overall pattern can be divided based on the recognition results of provincial low-carbon economic development pattern in China in long time series, which is convenient to clarify the provincial development positioning of different pattern types.

From Fig. 11, (a) the spillover type is mainly concentrated in the eastern coastal areas, including Beijing, Tianjin, Shanghai, Jiangsu, Zhejiang, Fujian, Shandong and Guangdong. (b) The main bridge type is mainly concentrated in the central area adjacent to the eastern coastal areas, including Anhui, Jiangxi, Hubei, Hunan, Chongqing, and Shaanxi. (c) The auxiliary bridge type is mainly concentrated in the middle and part of the southwest areas adjacent to the main bridge type, including Hebei, Shanxi, Liaoning, Henan, Guangxi, and Sichuan. (d) The beneficial type is mainly concentrated in the northeast, northwest, and part of southwest China, including Inner Mongolia, Jilin, Heilongjiang, Hainan, Guizhou, Yunnan, Gansu, Qinghai, Ningxia, and Xinjiang. This is consistent with the general direction of China's regional economic development strategy, such as first development of the eastern region, the rise of the central region, the development of the western region, and the revitalization of the northeast region, showing that the research results are scientific and reasonable.

Specifically, spillover provinces mainly radiate and drive the development of main bridge and auxiliary bridge provinces by giving play to the spatial spillover effect, especially the main bridge provinces with



Fig. 11. The overall pattern of China's provincial low-carbon economic development. 
small development capability gap, showing a stronger radiation effect. The main bridge type mainly plays the role of radiating and driving the provincial development of auxiliary bridge and beneficiary type, especially the auxiliary bridge type. And, the auxiliary bridge provinces further undertake the role of radiation driving the development of benefit provinces. Of course, in this process, the provinces of spillover and main bridge type, main bridge and auxiliary bridge type are easier to achieve the situation of "promoting development" through the flow and sharing of resource elements, but the strong radiation effect still mainly comes from the provinces with excellent low-carbon economic development capability.

It can be seen that the further improvement of China's global low-carbon economic development capability largely depends on the connecting role of bridge provinces, especially the main bridge provinces. This is mainly because regional economic development is a gradual process. It is difficult to produce strong spatial correlation between provinces with large gap in development capability, which limits the radiation driving effect. It is necessary to rely on the bridge support of provinces with medium and upper medium development capability to promote common development through the hierarchy of radiation effect. Of course, it is undeniable that the improvement of the low-carbon economic development capability of spillover provinces will help to improve the global lowcarbon economic development capability of China.

In the future, we should make full use of the radiation effects of spillover and main bridge provinces to improve the low-carbon economic development capability in auxiliary bridge provinces, to radiate and drive the development of benefit provinces, and finally achieve the development of China's global lowcarbon economy at a higher level. Concurrently, it is worth noting that Chongqing, Shaanxi and Sichuan, especially Chongqing with good low-carbon economic development capability, are expected to become a new growth pole in the western region. While Hebei, with its advantageous geographical location, is expected to become a new growth pole in the northeast region. Driven by the multi-center drive and the new regional growth pole, and taking advantage of the spatial linkage effect between provinces, it will build a new situation of joint and coordinated development across the region.

\section{Conclusions and Future Work}

The low-carbon economic development involves multi-dimensional elements. Concurrently, driven by the concept of common development of regional integration, the low-carbon economic development in a province is not only affected by its own development status, but also promoted and restricted by the spatial linkage with surrounding provinces. At this time, the research on pattern recognition based on attribute data of comprehensive evaluation value is not comprehensive enough. Concurrently, the undirectedunweighted network is difficult to truly reflect the inter-provincial detailed spatial correlation with directionality and weight, which is easy to lead to the inaccurate pattern recognition and development positioning of provincial low-carbon economic development. Therefore, based on the relationship data, this study takes $2008-2017$ as the research period, takes 30 China's provinces as the research objects, determines the inter-provincial spatial correlation strength of low-carbon economic development based on multi-dimensional gravity model, and constructs a directed-weighted complex network. Then, from three dimensions of overall, individual and group, the detailed spatial relationship between China's provinces is mined to recognize the low-carbon economic development pattern of different provinces and define the development orientation. The main conclusions are as follows.

(1) In terms of low-carbon economic development capability, during the study period, China's provincial low-carbon economic development capability has been continuously improved, but it shows an unbalanced development situation. The overall situation is generally from east to west, from strong to weak, and there is a jump pattern in some areas.

(2) In terms of correlation structure, the spatial correlation of China's provincial low-carbon economic development is increasingly close, but the spatial correlation strength between different provinces is quite different, showing a complex networked correlation structure with multiple characteristics such as multi-linearity, complexity, asymmetry and geographical proximity. It can be roughly divided into five regions: the eastern coastal areas including Beijing and Tianjin, centered on Hubei, Hunan and Jiangxi provinces in Central China and including the surrounding provinces, the northeast region, the northwest region and the southwest region. This is in line with China's regional economic development pattern and development strategy.

(3) In terms of network center, the network center of the complex network of China's provincial low-carbon economic development shows a certain degree of solidification. Among them, the provinces in the central region are at the center of the complex network, and the provinces in the western region are at the edge of the network. It is worth noting that although Beijing, Tianjin, Shanghai, and Guangdong, with superior low-carbon economic development capability, are easy to have a strong radiation driving effect on other provinces, due to the large development gap between them, it is difficult to receive the radiation effect from them equally, which makes the central position in the complex network not prominent enough. On the contrary, Hebei, 
with the advantage of its connection location in the middle and eastern region, and maintaining a relatively similar development pattern with most provinces, is easy to have a two-way correlation between radiation and reception with them, and occupies an important position in the complex network. Notably, the importance of the location in the network depends on its own development capability, and is inseparable from its superior geographical location, concurrently, maintains a relatively similar development pattern with the surrounding provinces.

(4) In terms of cohesive subgroups, the cohesion process of different provinces in the network is characterized by variability and geographical proximity. However, during the study period, the overall cohesion direction of the network has not changed greatly, and finally, it will stabilize the cohesion between provinces with similar low-carbon economic development level and geographical proximity.

(5) In terms of low-carbon economic development patterns, on the whole, China's provincial lowcarbon economic development pattern has slight changes, but generally relatively stable, which can be roughly divided into four types: spillover, main bridge, auxiliary bridge, and benefit type. Among them, the spillover provinces are concentrated in the eastern coastal areas, the bridge provinces are concentrated in the central and surrounding southwest areas adjacent to the spillover provinces, while the benefit provinces are concentrated in the northeast, northwest and some southwest areas. Notably, Chongqing, Shaanxi, Sichuan and Hebei are expected to become new growth poles of benefit provinces in the low-carbon economic development. In the future, we should make full use of the radiation effects of spillover and bridge provinces, improve the low-carbon economic development capability in auxiliary bridge provinces, and then radiate and drive the development of benefit provinces. It is expected to build a situation of coordinated development of China's global low-carbon economy at a higher level. Relevant provincial government departments should break the inter-provincial administrative boundaries, create more spatial correlation effects, to give full play to the power source role of spillover provinces and the bridge support role of bridge provinces.

On the whole, the research results of low-carbon economic development capability, spatial correlation structure and overall development pattern are consistent with the existing relevant literature, and are consistent with the regional development strategies such as first development of the eastern region, the rise of the central region, the development of the western region, and the revitalization of the northeast region. Findings in this study could provide a scientific basis for fine and differentiated policy-making related to China's global economic low-carbon sustainable development. However, it should be pointed out that due to the limitation of data availability, based on the provincial research scale, this study can only describe the detailed spatial correlation of China's provincial low-carbon economic development from the macro level. Future research could conduct quantitative analysis from the meso and micro scale, to guide the formulation of refined and differentiated low-carbon economic development strategies.

\section{Acknowledgments}

The work was supported by the Fund project of Guangdong Key Laboratory of urban perception, monitoring and early warning enterprises (Grant No. 2020b121202019), and National Natural Science Foundation of China (Grant No. 41301377).

\section{Conflict of Interest}

The authors declare no conflict of interest.

\section{References}

1. WU R.W., DONG J.F., ZHOU L., ZHANG L. Regional Distribution of Carbon Intensity and its Driving Factors in China: An Empirical Study Based on Provincial Data. Polish Journal of Environmental Studies, 27 (3), 1331, 2018.

2. LI C., LI X.H., ZHU X.G. Examining Economic Development and Carbon Emissions in China's LowCarbon Pilot Provinces. Polish Journal of Environmental Studies, 26 (6), 2619, 2017.

3. WU L.P., ZHU Q.Y. Impacts of the carbon emission trading system on China's carbon emission peak: a new data-driven approach. Natural Hazards, 107, 2487, 2021.

4. CHEN X., SHUAI C.Y., WU Y., ZHANG Y. Analysis on the carbon emission peaks of China's industrial, building, transport, and agricultural sectors. Science of the Total Environment, 709, 135768, 2020.

5. Energy White Paper: Our Energy Future - Creating a Low Carbon Economy. UK Department of Trade and Industry: London, 2003

6. MA D.L. China's Low Carbon Economic Growth Efficiency: an Analysis Involving Carbon Sink. Polish Journal of Environmental Studies, 26 (3), 1147, 2017.

7. CHEN Y.Z., DUAN L.L., Low-Carbon Economy \& LowCarbon City: China's Inevitable Choice for Sustainable Economic Development. Sci Res Publ, Inc-Srp: Irvin, 479, 2012

8. ZHENG L.C., FU J.F., LI J.S. Evaluation on the development level and spatial process of provincial lowcarbon economy in China. China Population, Resources and Environment, 21 (07), 80, 2011.

9. PAN W.Y., GULZAR M.A., HASSAN W. Synthetic Evaluation of China's Regional Low-Carbon Economy Challenges by Driver-Pressure-State-Impact-Response Model. International Journal of Environmental Research and Public Health, 17 (15), 5463, 2020.

10. ALPTEKIN O., ALPTEKIN N., SARAC B. Evaluation of Low Carbon Development of European Union Countries 
and Turkey Using Grey Relational Analysis. Tehnicki Vjesnik-Technical Gazette, 25 (5), 1497, 2018.

11. PENG T., DENG H.W. Research on the sustainable development process of low-carbon pilot cities: the case study of Guiyang, a low-carbon pilot city in south-west China. Environment Development and Sustainability, 23 (2), 2382, 2021.

12. SHI L.Y., XIANG X.Q., ZHU W., GAO L.J. Standardization of the Evaluation Index System for Low-Carbon Cities in China: A Case Study of Xiamen. Sustainability, 10 (10), 2018.

13. QU Y., LIU Y. Evaluating the low-carbon development of urban China. Environment Development and Sustainability, 19 (3), 939, 2017.

14. WANG Y.A., FANG X.L., YIN S.W., CHEN W. Lowcarbon development quality of cities in China: Evaluation and obstacle analysis. Sustainable Cities and Society, 64 (9), 102553, 2021.

15. LI S.L., LEI M. Evaluation of provincial low-carbon economic development based on TOPSIS and its spatial panel measurement analysis. Chinese Journal of Management Science, 22 (S1), 741, 2014.

16. YANG Z. Comprehensive evaluation of China's lowcarbon economy development level. Master's Thesis, Liaoning University, Liaoning, China, 2013.

17. YANG X., LI R.R. Investigating low-carbon city: Empirical study of Shanghai. Sustainability, 10 (4), 2018.

18. DUAN Y., MU H.L., LI N., LI L.L., XUE Z.Q. Research on comprehensive evaluation of low carbon economy development level based on AHP-Entropy method: A case study of Dalian. Clean Energy for Clean City: Cue 2016 - Applied Energy Symposium and Forum: Low-Carbon Cities and Urban Energy Systems, 104, 468, 2016.

19. SHI X.F., SUN Y., CUI Y. Evaluation of Tianjin's lowcarbon economic development level based on entropyprincipal component analysis. Science and Technology Management Research, 38 (03), 247, 2018.

20. DU H.B., CHEN Z.N., MAO G.Z., LI M.Y.R., CHAI L.H. A spatio-temporal analysis of low carbon development in China's30 provinces: A perspective on the maximum flux principle. Ecological Indicators, 90, 54, 2018.

21. SUN J.W., YAO P. Evaluation of low carbon economic development level and regional comparative analysis: A case study of Xinjiang. Areal Research and Development, 33 (3), 127, 2014 [In Chinese].

22. BAI C.Q., ZHOU L., XIA M.L., FENG C. Analysis of the spatial association network structure of China's transportation carbon emissions and its driving factors. Journal of Environmental Management, 253 (Jan.1), 109765.1-109765.12, 2020.

23. MA Y., XUE F. Deciphering the Spatial Structures of City Networks in the Economic Zone of the West Side of the Taiwan Strait Through the Lens of Functional and Innovation Networks. Sustainability, 11 (10), 2975, 2019.

24. QI W., XIONG H.Y., PENG X.Y. How to Promote International Competitiveness of China's Renewable Energy Products? - Based on SNA Theory Polish Journal of Environmental Studies, 30 (5), 4163, 2021.

25. YU G.H., HE D.Y., LIN W.L., WU Q.H., XIAO J.X., LEI X.F., XIE Z.Q., WU R.J. China's Spatial Economic Network and Its Influencing Factors. Complexity, 2020 (2020), 2020.

26. SUN Q.P., WANG S.J., ZHANG K.Q., MA F., GUO X.Z., LI T.Z. Spatial Pattern of Urban System Based on Gravity Model and Whole Network Analysis in Eight Urban Agglomerations of China. Mathematical Problems in Engineering, 2019, 2019.

27. HU W., HU Y.W., WANG C.L., CHEN X.Z. Construction and analysis of China's industrial network of carbon correlation. Applied Ecology and Environmental Research, 17 (6), 14487, 2019.

28. HE Y.Y., WEI Z.X., LIU G.Q., ZHOU P. Spatial network analysis of carbon emissions from the electricity sector in China. Journal of Cleaner Production, 262, 121193, 2020.

29. WANG F., GAO M.N., LIU J., FAN W.N. The Spatial Network Structure of China's Regional Carbon Emissions and Its Network Effect. Energies, 11 (10), 2706, 2018.

30. ZHAO Y.P. An empirical study on the impact of urban agglomeration economic connection network on economic growth. Master's Thesis, East China University of Political Science and Law, Shanghai, China, 2019.

31. GAN C., VODA M.H., WANG K., CHEN L.J., YE J. Spatial network structure of the tourism economy in urban agglomeration: A social network analysis. Journal of Hospitality and Tourism Management, 47 (6), 124, 2021.

32. HAN R.B., CAO H.H., LIU Z.W. Re-exploration of China's urban system based on comprehensive gravity model. Scientia Sinica (Terrae). 48, 1670, 2018.

33. WANG F., LU Y., LI J., NI J. Evaluating Environmentally Sustainable Development Based on the PSR Framework and Variable Weigh Analytic Hierarchy Process. International Journal of Environmental Research and Public Health, 18 (6), 2021.

34. WANG Q., LI S.Q., LI R.R. Evaluating water resource sustainability in Beijing, China: Combining PSR model and matter-element extension method. Journal of Cleaner Production, 206, 171, 2019.

35. GUO Y.J. A new dynamic comprehensive evaluation method. Journal of Management Sciences in China., 2002 (02), 49-54, 2002.

36. WANG C.K., GONG Z.W. Modification of the index standardization method in the "vertical and horizontal" unfolding grade method. Statistics \& Decision., 02, 77, 2016.

37. G.H. Z., JIA P., ZHOU A.M. Improved degree centrality in directed weighted networks. Journal of Computer Applications, 40 (S1), 141, 2020. 